\title{
MATRIX ANALYSIS OF ECG PARAMETERS MAY BE A WAY TO IMPROVE QUALITY OF FUNCTIONAL STATE MONITORING DURING EXERCISING
}

\author{
Jonas Poderys ${ }^{1}$, Liepa Bikulčienè ${ }^{2}$, Eugenijus Trinkūnass ${ }^{1}$ Kristina Poderienè $\dot{e}^{1}$, \\ Alfonsas Buliuolis ${ }^{1}$, Alfonsas Vainoras ${ }^{3}$ \\ Lithuanian Sports University, Kaunas, Lithuania \\ Kaunas University of Technology², Kaunas, Lithuania \\ Lithuanian University of Health Sciences ${ }^{3}$, Kaunas, Lithuania
}

\begin{abstract}
Research background. The aim of this study was to compare the peculiarities of dynamics of cardiovascular functional state indices during bicycle ergometry by applying the steep or slow increase in workload.

Methods. Twenty five males attending health promotion sport clubs took part in two cardiovascular testing procedures performing graded exercise stress, i.e. increasing the workload every min, and increasing the workload every 6 min. They exercised till the inability to continue the task or when distressing cardiovascular symptoms supervened. 12 Lead ECG was recovered and analysed.

Results. The steep increase of registered cardiovascular parameters was observed at onset of exercising and the rate of it depended on the increase rate in workload. Registered maximal changes of heart rate, JT interval, and the ratio JT/RR as a functional state index at the moment of refusing to continue the graded exercise test were of the same level while applying the steep or slow increase in workload.

Conclusions. The rate of increase in workload determines the mobilization rate but not the degree of increase of ECG parameters. Maximal changes of heart rate, JT interval, and the ratio JT/RR as functional state index at the moment of refusing to continue the graded exercise test were the same level while applying the steep or slow increase in workload.
\end{abstract}

Keywords: cardiovascular system, graded exercise stress, functional state.

\section{INTRODUCTION}

$\mathrm{F}$ eedback while exercising is a way to achieve the effect of health promotion. Various types of heart rate (HR) monitors were widely used for over 30 years (Achten \& Jeukendrup, 2003). But training session has two purposes at least. Firstly, the workload should impact appropriate internal body changes and secondly, the workload should not overreach individual physiological abilities of patient by creating the risk for his or her health. This problem cannot be solved only by monitoring HR (Gademan et al., 2012) and requires new modern solutions.
The complex system model of fatigue suggests that activity during exercise occurs in an integrative manner, where internal signals from a number of different physiological systems, which are in a constant state of flux, are used by an integrative "governor system" to continuously modulate exercise by regulating power output, and therefore pacing strategy, to levels appropriate to the capacity of each different physiological system relative to the level of intensity of the fatiguing exercise being performed (Gibson, 2008). Each adjustment in power output results in changes to all physiological systems, and therefore a specific 
period of time is required for afferent information to be able to assess the result of these changes in the physiological systems that will enable further adjustments in power output. This time lag requirement creates fluctuations in power output and in the different physiological systems (Gibson, 2008; Torrents \& Balague, 2006).

Analytical approach has been successful in describing the physical world but it is not the same when we have to handle the treatment or couching related issues, i.e. by applying it to living objects (Hristovski, Venskaityte, Vainoras, Balague, \& Vazquez, 2010; Mayer-Kress, 2001; Torrents \& Balague, 2006). Human body during exercising in fact represents a much more complex phenomenon reflecting the nonlinear processes of body's general functional state and its fatigability in a fractal or chaotic manner (Hristovski et al., 2010; Torrents \& Balague, 2006; Tulppo et al., 2001). Interrelation mechanisms of body systems and components' interactions are essential in determining how body functions as a whole as a complex dynamic adaptive system. The aim of this study was to complement an analytical approach by new methodology of data sequences analysis of cardiovascular indices under conditions of increasing fatigue.

\section{METHODS}

The participants of the study were 10 healthy adult males attending health promotion sport, and two elite athletes. One of these athletes was a leader in long distance running and a winner a lot of races this year and other athlete shortly stopped the intensive training because of the medical diagnosis "Overtraining".

Each participant had spent 20 min seated and after registration of the base-line values of ECG they underwent a $50 \mathrm{~W}$ increase in workload every $6 \mathrm{~min}$ (60 revolutions $/ \mathrm{min})$, i.e. graded exercise test (bicycle ergometry) and they exercised till the inability to continue the task or unless distressing cardiovascular symptoms supervened. The ECG registered continuously during the exercising and first 5 min ofrecovery. The RR interval, i.e., heartrate (HR), duration of QRS and ST-segment depression were taken for further analysis. For comparison of different processes the new diagnostic technology was created. Those technologies have a possibility to get maximal amount of diagnostic information by using minimal amount of recorded processes. The software performs in real time data preprocessing (noise reduction, artefact suppression) and recognition of parameters from continuously registered ECG.

Theoretical background. For investigation of two objects interaction two synchronous numerical time series $\left(x_{n} ; n=0,1,2, \ldots\right)$ and $\left(y_{n} ; n=0,1,2, \ldots\right)$ representing exploratory object must be formed. Here $x_{n}$ and $y_{n}$ are real numbers and they represent results of some measurements. Usually it is electrocardiogram signals (or some parameters of signals) of one or two associated persons. The statistical time series investigation methods are very popular and in this case elements of time series are random variables (Dahlhaus, Kurths, Maass, \& Timmer, 2008). When elements of series are determined variables, information about object of investigation can be described using mathematical relationships (Arnold, 1998). In this paper the method based on matrix theory is proposed.

Let two numerical time series $\left(x_{n} ; n=0,1,2, \ldots\right)$ and $\left(y_{n} ; n=0,1,2, \ldots\right)$ be given. Then the matrix time series $\left(A_{n} ; n=0,1,2, \ldots\right)$ can be formed. Here $A_{n}:=\left[\begin{array}{ll}a_{n} & b_{n} \\ c_{n} & d_{n}\end{array}\right]$ and coefficients $a_{n}:=x_{n}$, $b_{n}:=\alpha\left(x_{n-1}-y_{n-1}\right), c_{n}:=\beta\left(x_{n+1}-y_{n+1}\right), d_{n}:=y_{n}$, when parameters $\alpha, \beta$ are at choice dependent on properties of time series $\left(x_{n} ; n=0,1,2, \ldots\right)$, $\left(y_{n} ; n=0,1,2, \ldots\right)$. In the simplest case, coefficients are $\alpha=\beta=1$.

So, in this case four time series $\left(a_{n} ; n=0,1,2, \ldots\right), \ldots \quad\left(d_{n} ; n=0,1,2, \ldots\right)$ and one matrix time series $\left(A_{n} ; n=1,2,3, \ldots\right)$ are obtained. Of course these series can be formed using other mathematical relationships. Time series scheme is shown in Figure 1.

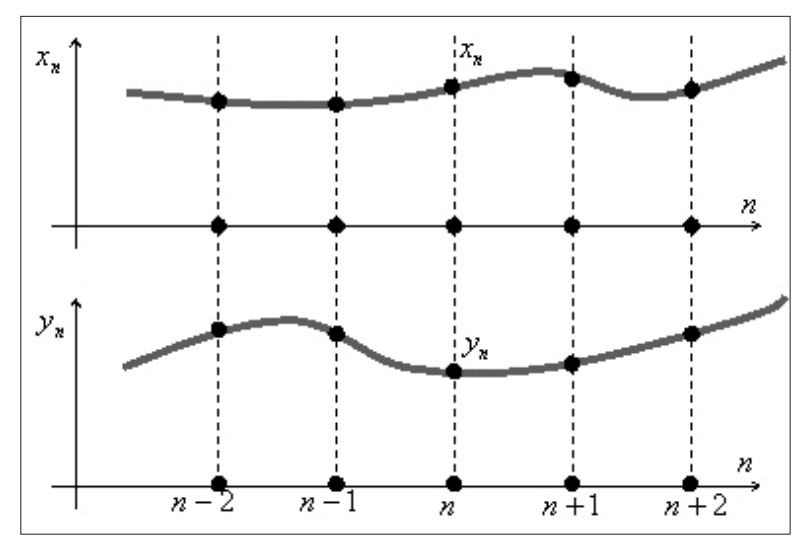

Figure 1. The scheme of time series

Different methods for analysis of obtained series can be used. In this investigation of matrix 
time series the numerical characteristics of second order matrices and main components of matrices $A_{n}$ were used:

1. $\operatorname{Tr} A_{n}:=a_{n}+d_{n}$ (trace of matrix $\left.A_{n}\right)$,

2. $\operatorname{dfr} A_{n}:=a_{n}-d_{n}$ (difference),

3. $\operatorname{cdp} A_{n}:=b_{n} \cdot c_{n}$ (co-diagonal product),

4. $B_{n}:=\left[\begin{array}{cc}\frac{\mathrm{dfr} A_{n}}{2} & b_{n} \\ c_{n} & -\frac{\mathrm{dfr} A_{n}}{2}\end{array}\right]$ (main component of matrix $A_{n}$ ).

From these initial parameters follow characteristics which have more applicative sense:

5. $\operatorname{dsk} A_{n}=\left(\operatorname{dfr} A_{n}\right)^{2}+4 \operatorname{cdp} A_{n}$ (discriminate)

6. $\operatorname{det} A_{n}=\frac{1}{4}\left(\left(\operatorname{Tr} A_{n}\right)^{2}-\operatorname{dsk} A_{n}\right)($ determinant $)$,

7. $\lambda_{1,2}=\frac{1}{2}\left(\operatorname{Tr} A_{n} \pm \sqrt{\mathrm{dsk} A_{n}}\right) \quad$ (eigenvalues matrix $A_{n}$ ),

Two important types of matrices in matrix analysis are important: the matrix $I$ is called idempotent (matrix of stable power), if $I^{2}=I$ and the $N$ - nilpotent (matrix losing power), if $N^{2}=\mathbf{0}$, where $\mathbf{0}$ is zero matrix.

If discriminates of matrices $A_{n}$ become to zero then matrices $A_{n}$ from idempotent become to nilpotent. Mathematically, if the limits $\operatorname{dsk} A_{n} \rightarrow 0$, $b_{n} \rightarrow \bar{b}, c_{n} \rightarrow \bar{c}$, satisfying condition $\bar{b} \cdot \bar{c} \leq 0$ exist, then $B_{n} \rightarrow\left[\begin{array}{cc} \pm \sqrt{|\bar{b} \cdot \bar{c}|} & \bar{b} \\ \bar{c} & \mp \sqrt{|\bar{b} \cdot \bar{c}|}\end{array}\right]:=\bar{B}$, and $\bar{B}^{2}=\mathbf{0}$.

It shows that chosen time series $\left(x_{n} ; n=0,1,2, \ldots\right)$ and $\left(y_{n} ; n=0,1,2, \ldots\right)$ become similar and describe more associated system. The sequence of idempotent matrices $\left(\sqrt{\operatorname{dsk} A_{n}} \cdot I_{n} ; n=0,1,2, \ldots\right)$ if the limit transitions $\left|\lambda_{n}-\mu_{n}\right| \rightarrow 0, \sqrt{\operatorname{dsk} A_{n}} \cdot I_{n} \rightarrow \bar{B}$ exists can be formed, and this sequence shows evolution of matrix sequence $\left(A_{n} ; n=0,1,2, \ldots\right)$.

From definitions of matrix characteristics the main interest have discriminates of matrices $A_{n}$, accordingly the time series $\left(\operatorname{dsk} A_{n} ; n=0,1,2, \ldots\right)$ investigation is important.

The elements of matrices can be formed in more complicated way:

$$
\begin{aligned}
& b_{n}:=\alpha_{1}\left(x_{n+1}-y_{n+1}\right)+\alpha_{2}\left(x_{n+2}-y_{n+2}\right), \\
& c_{n}:=\beta_{1}\left(x_{n-2}-y_{n-2}\right)+\alpha_{2}\left(x_{n-1}-y_{n-1}\right) .
\end{aligned}
$$

Here $\alpha_{1}+\alpha_{2}=1, \alpha_{1}>\alpha_{2}>0, \quad \beta_{1}+\beta_{2}=1$, $\beta_{1}>\beta_{2}>0$.

On purpose to escape noise influence, the elements can be averaging. Then terms of time matrices $\quad a_{n}:=\sum_{j=-k}^{l} \gamma_{j}^{\prime} x_{n+j}, \quad d_{n}:=\sum_{j=-k}^{l} \gamma_{j}^{\prime \prime} y_{n+j}$, $b_{n}:=\sum_{j=1}^{l} \sigma_{j}^{\prime}\left(x_{n+j}-y_{n+j}\right), c_{n}:=\sum_{j=-k}^{-1} \sigma_{j}^{\prime \prime}\left(x_{n+j}-y_{n+j}\right)$.

If number of terms in sums increases, the sequence of discriminates become smoother, but its character is the same. From numerical investigation it is obtained that changing of parameters $\alpha, \beta$ has influence only to amplitude of sequence, but not for character, because in further calculations the simplest case of matrices formation were used. The initial data was normalized using formula

$$
x_{\text {new value }}=\frac{x_{\text {old value }}-x_{\min }}{x_{\max }-x_{\min }},
$$

where $x_{\min }$ and $x_{\max }$ are minimal and maximal physiological values of parameter.

\section{RESULTS}

Working capacity. The task for the participants of this study was to continue exercising as much as they can, i.e. till the inability to continue it. All participants of the study were able to perform the workload up to 17 minute (workload 150W) and the highest performance in non-athletes cohort demonstrated by one participant was 27 minutes (the stage of $250 \mathrm{~W}$ ). The averaged data of performance abilities can be depicted by mentioning that nonathletes were able to continue the task of increasing workload for $23.5 \pm 2.4 \mathrm{~min}$. The arrows with figures in Figure 2 show the number of participants who were able further to continue the increasing workload.

The elite athletes demonstrated the better muscular performance, i.e. they were able to continue the increasing workload up to 35 th minute (first athlete) and up to 31.5th minute (second athlete).

HR changes. The HR changes were related to the increase in workout as it was shown in Figure 2 or in Figures $3-6$ where RR intervals of ECG were presented in grey (background of figure). No one of all these cases can reveal the transition of body states such as anaerobic threshold or when the workload became not physiological. Gradually the increase of HR without steep changes can reveal if 
the essential and important body changes occurred or if it is difficult to say at what moment they did.

Discriminant changes. The matrix analysis of the ECG parameters has the aim to more precisely answer the question if discriminate changes call reveal the essential changes of functional state while exercising, i.e. under the conditions of increasing fatigue. Aiming to answer this question the analysis of individual cases could be useful for analysis.

Figure 3 presents the dynamics of discrimination between RR intervals and ST-segment depression during incremental exercise test and recovery. As we can see form Figure 3, the discriminant changes go parallel with the HR changes.

The different dynamics of discrimination between RR intervals and QRS during incremental exercise test were found when the data of two athletes runners were compared (Figure 3). The essential difference between these two cases was that discriminant values of the second athlete with medical diagnosis "Overtraining" were significantly higher during exercising (Figure $3-\mathrm{A})$. The first elite athlete not only was able to demonstrate better muscular performance, i.e. continue exercising longer, but the discriminant values were low and only at the end of exercising the discriminant values showed a steep increase (Figure 3-B).

Figure 5 presents the dynamics of discrimination between the amplitude of $\mathrm{R}$ wave and RR intervals during incremental exercise test and recovery of elite athlete. What was important about the the dynamics of discrimination during exercising under the conditions of increasing fatigue, that a steep change of discriminant values was observed in the middle of the exercise test.
Figure 2. Dynamics of HR during incremental exercise test and recovery
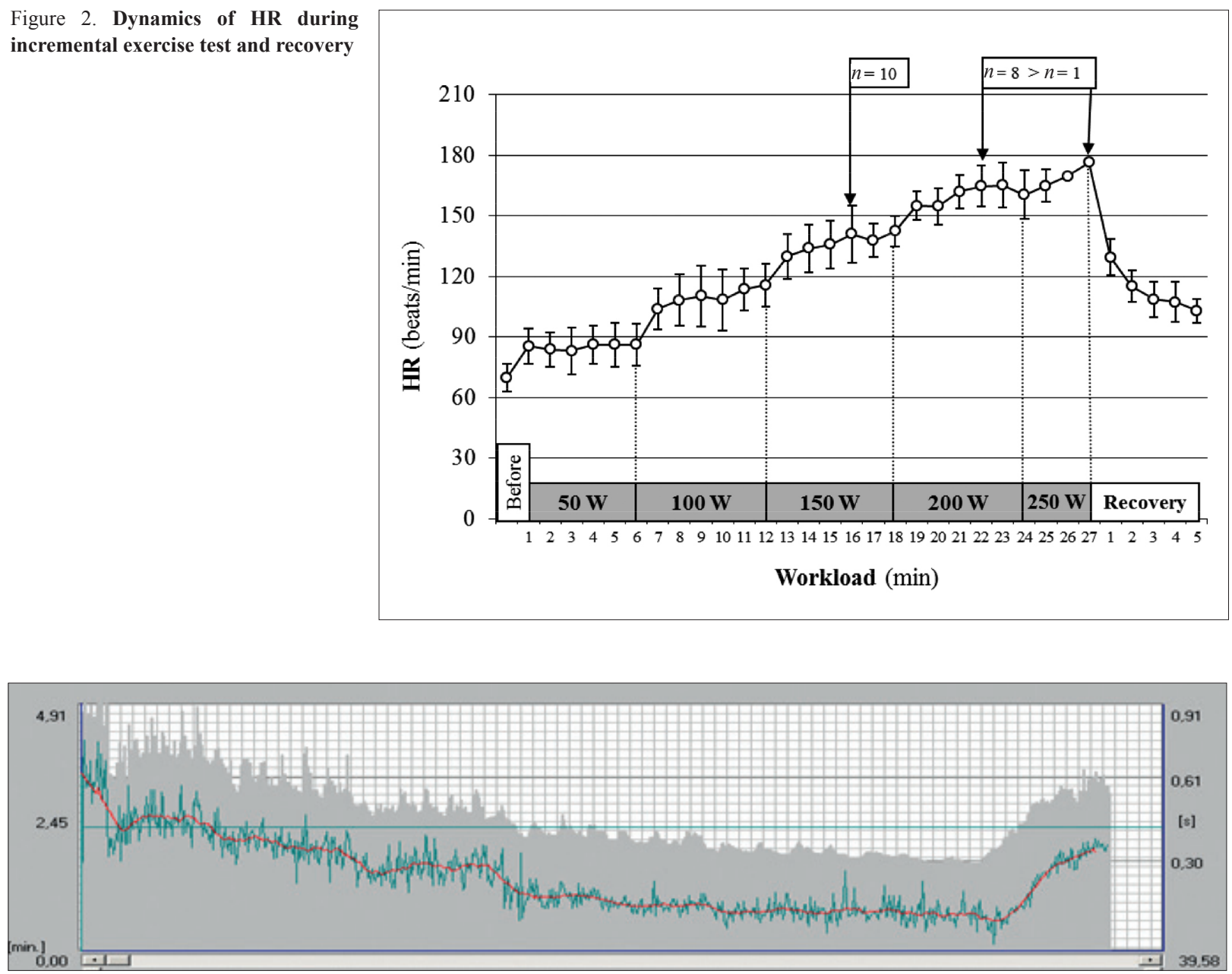

Figure 3. Dynamics of discriminants between RR intervals and ST-segment depression during incremental exercise test and recovery

Notes. $\mathbf{X}$ axis - discriminant value (left); RR intervals, s (right). Y axis - time, min

Grey (background) shows dynamics of RR intervals of ECG;

Green line-discriminant; red line-discriminant averaged of 10 figures. 


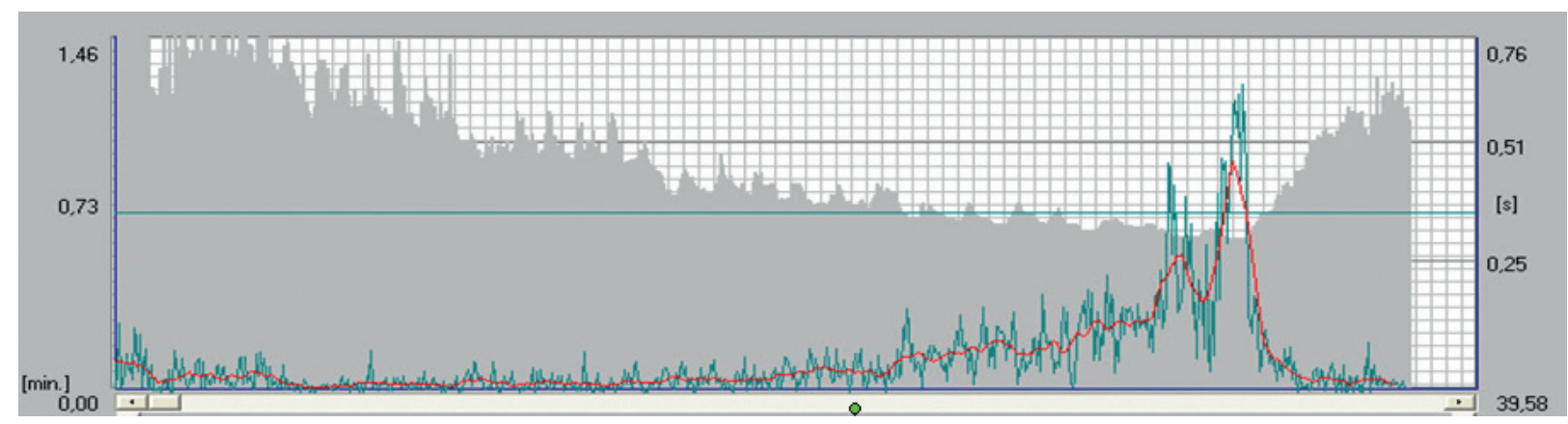

A

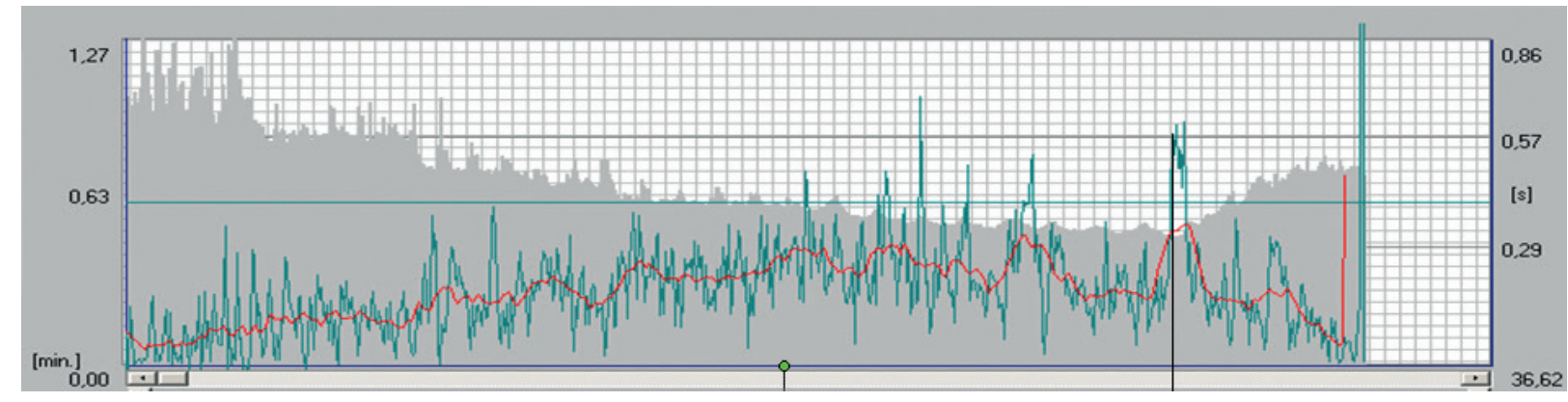

B

Figure 4. Dynamics of discriminants between RR intervals and QRS during incremental exercise test and recovery of elite athlete (A) and other athlete with medical diagnosis "Overtraining”(B).

Notes. $\mathbf{X}$ axis - discriminant value (left); RR intervals, s (right). Y axis - time, min

Grey (background) shows dynamics of RR intervals of ECG;

Green line-discriminant; red line-discriminant averaged of 10 figures.

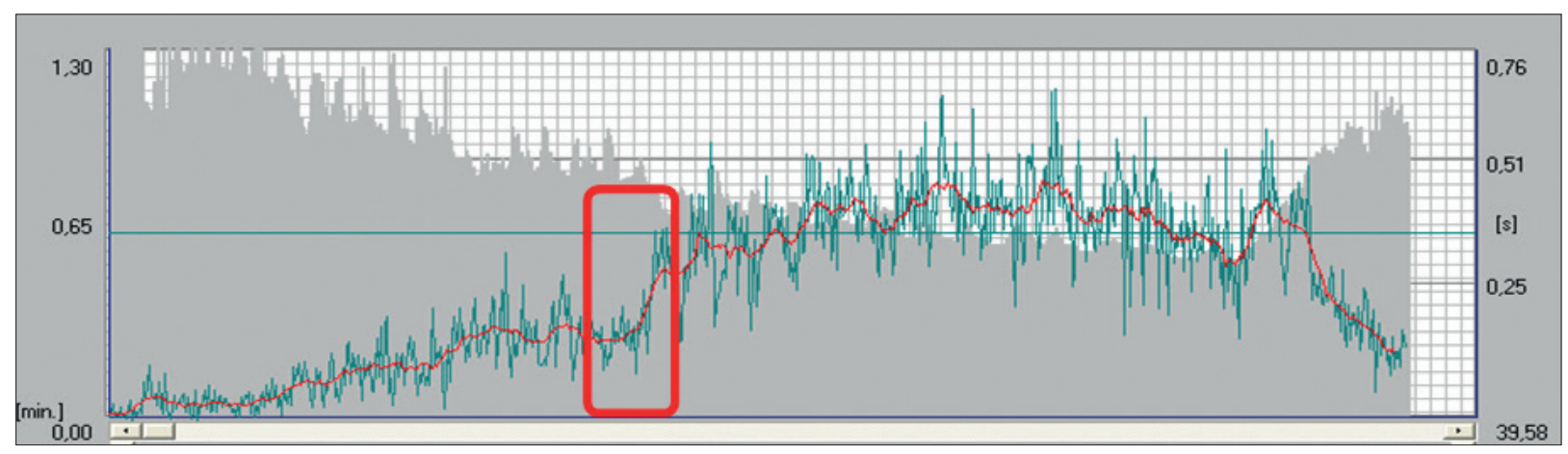

Figure 5. Dynamics of discriminants between amplitude of $\mathbf{R}$ wave and $\mathbf{R}$ intervals during incremental exercise test and recovery of elite athlete

Notes. $\mathbf{X}$ axis - discriminant value (left); RR intervals, s (right). $\mathbf{Y}$ axis - time, min Grey (background) shows dynamics of RR intervals of ECG;

Green line-discriminant; red line-discriminant averaged of 10 figures.

\section{DISCUSSION}

Exercise dosage control and feedback during exercising is a relevant problem that required a new modern solution. There are various methods of measuring the body reactions to workloads and physical exertion, but their applicability and value are widely discussed and analysed (Boettger et al., 2010). Functional state could be recognized by assessing the reactions to exercising workload
(Mailey et al., 2010), but the question about indices and criteria for assessment and formation of feedback remains on agenda of scientific studies.

At onset of exercise all body systems adapt with a series integrated responses to meet the metabolic demands of exercising muscles (Enoka \& Duchateau, 2008; Hughson \& Tschakovsky, 1999; Vainoras, 2002). During exercising all levels of body structure, i.e. sub cellular, cellular, tissue, organs, systems are activated and all physiological systems 
appear to show similar non-monotonic activity at all times and at all functional levels (Gibson, 2008). For investigation of these peculiarities in time, various mathematical methods are applied in order to estimate a human like a complex system (Costa, Peng, \& Goldberger, 2008; Gademan et al., 2012). The human body is a complex system. Particularly, it is possible to assess the functional peculiarities while investigating elements of this system and its relationships (Hristovski et al., 2010). Fatigue arises through the interaction of the component processes and causes the reduction of low activity in the neurobiological system. Although specific physiological mechanisms are probably highly task-dependent (Enoka \& Duchateau, 2008), there is the non-linear dynamic system theory that enables to indicate specific causes of fatigue and provides knowledge of the phenomenon of critical instability. Lately, an integrative point of view on the process of fatigue suggests that the origin of the fatigue or optimal functional state is related to the interaction between the physiological systems, between various physiological mechanisms.

Different parameters of ECG can describe various physiological processes. According to the model of "Integral assessment of body during exercising" (Vainoras, 2002), HR changes can be treated as a regulatory signal requiring changes in the activity of cardiovascular response; ST-segment depression as indicator of functional ischemic episodes in cardiac musculature (Gademan et al., 2012; Guldenring et al., 2012; Jernberg, Lindahl, \& Wallentin, 1999; Taglieri et al., 2011) can be treated as a metabolic signal. The results obtained during matrix analysis showed that discrimination between RR intervals and ST-segment depression during incremental exercise test and recovery was parallel to the HR changes (Figure 3). It can be concluded that regulatory signals are strictly interconnected with the cardiac metabolism. If we want to follow the model "Integral assessment of body during exercising", the QRS complex of ECG can be treated as a signal for the contraction of cardiac musculature. The small values of discrimination between RR intervals and QRS during incremental exercise test of elite athlete (Figure $3 \mathbf{A}$ ) indicate a good adjustment between the regulatory signals of the system and organ levels, and the steep increase of discriminants at the end of incremental exercise test indicate the concatenation between these fractal levels of the body was lost.
The actual problem is how to catch a small but essential change in functional state of the body during the training session. Some intensity of exercising is physiological and impacts positive body changes, but when the intensity of exercising exceeds the physiological limits, the continuation of exercising is damaging. Monitoring HR cannot find and reveal such important points, e.g. at what moments the body responses to exercise workout become not physiological. It is difficult to handle the data obtained by matrix analysis of ECG parameters because there not a lot such studies. Studies designed to analyse the peculiarities of dynamics of cardiovascular, muscular or other physiological indices during exercising point out that sudden change of discriminant values indicate the internal reorganization within the physiological mechanisms. Figure 5 showed the dynamics of discrimination between the amplitude of $\mathrm{R}$ wave and RR intervals during incremental exercise test. The characteristic feature in this dynamics was a sudden increase of discrimination at some moment of increasing workload. There were no significant changes in HR dynamics at this moment. So we can state that the matrix analysis, i.e. concatenation between ECG parameters, can be more sensitive to define the critical changes within the body. The more detailed studies are needed to find out what ECG parameters or cardiovascular parameters are more useful for outlining the dynamics of internal body changes during exercising for health purposes or during elite training sessions.

\section{CONCLUSION}

It is difficult to make a decision about physiologically suitable intensity of exercising or at what a moment exercising becomes not physiological and damaging. Individual assessment the dynamics of concatenation between ECG parameters suggests the usefulness of such approach in the field of functional state monitoring and feedback formation during exercising. Matrix analysis of ECG parameters may be a way to improve the quality of functional state monitoring during exercising.

Acknowledgement. This research has been supported by ITEA 2 Programme, Project CareWare, No. 13034. 


\section{REFERENCES}

Achten, J., \& Jeukendrup, A. E. (2003). Heart rate monitoring: Applications and limitations. Sports Medicine, 33(7), 517-538.

Arnold, D. (1998). Methods in the theory of time series analysis. Fundamental Principles of Mathematical Science (Vol. 1430). New York: Springer-Verlag.

Boettger, S., Puta, C., Yeragani, V. K., Donath, L., Muller, H. J., Gabriel, H. H., \& Bar, K. J. (2010). Heart rate variability, QT variability, and electrodermal activity during exercise. Medicine and Science in Sports and Exercise, 42(3), 443-448. doi: 10.1249/ MSS.0b013e3181b64db1

Costa, M. D., Peng, C. K., \& Goldberger, A. L. (2008). Multiscale analysis of heart rate dynamics: entropy and time irreversibility measures. Cardiovascular Engineering, 8(2), 88-93. doi: 10.1007/s10558-0079049-1

Dahlhaus, R., Kurths, J., Maass, P., \& Timmer, J. (2008). Mathematical methods in time series analysis and digital image processing (Understanding complex systems series). Springer Verlag.

Enoka, R. M., \& Duchateau, J. (2008). Muscle fatigue: What, why and how it influences muscle function. The Journal of Physiology, 586(1), 11-23. doi: 10.1113/ jphysiol.2007.139477

Gademan, M. G., Uberoi, A., Le, V. V., Mandic, S., van Oort, E. R., Myers, J., \& Froelicher, V. F. (2012). The effect of sport on computerized electrocardiogram measurements in college athletes. European Journal of Preventive Cardiology, 19(1), 126-138. doi: $10.1177 / 1741826710392669$

Gibson, A. S. C. (2008). Complex System Control of Activity During Fatiguing Exercise. 2nd International Congress of Complex Systems in Sport (2nd ICCSS), 10th European Workshop of Ecological Psychology (10th EWEP). Madeira, Portugal.

Guldenring, D., Finlay, D. D., Nelwan, S. P., Nugent, C. D., Donnelly, M. P., \& Bond, R. R. (2012). Estimation performance of a reduced lead system during continuous 12-lead ECG ST-segment monitoring. Journal of Electrocardiology, 45(6), 604-608. doi: 10.1016/j.jelectrocard.2012.08.012
Hristovski, R., Venskaityte, E., Vainoras, A., Balague, N., \& Vazquez, P. (2010). Constraints-controlled metastable dynamics of exercise-induced psychobiological adaptation. Medicina, 46(7), 447-453.

Hughson, R. L., \& Tschakovsky, M. E. (1999). Cardiovascular dynamics at the onset of exercise. Medicine and Science in Sports and Exercise, 31(7), 1005-1010.

Jernberg, T., Lindahl, B., \& Wallentin, L. (1999). STsegment monitoring with continuous 12-lead ECG improves early risk stratification in patients with chest pain and ECG nondiagnostic of acute myocardial infarction. Journal of the American College of Cardiology, 34(5), 1413-1419.

Mailey, E. L., White, S. M., Wojcicki, T. R., Szabo, A. N., Kramer, A. F., \& McAuley, E. (2010). Construct validation of a non-exercise measure of cardiorespiratory fitness in older adults. BMC Public Health, 10, 59. doi: 10.1186/1471-2458-10-59.

Mayer-Kress, G. J. (2001). Complex systems as fundamental theory of sports coaching? Retrieved from http://arxiv.org/html/nlin/0111009).

Taglieri, N., Marzocchi, A., Saia, F., Marrozzini, C., Palmerini, T., Ortolani, P., . . Rapezzi, C. (2011). Shortand long-term prognostic significance of ST-segment elevation in lead aVR in patients with non-ST-segment elevation acute coronary syndrome. The American Journal of Cardiology, 108(1), 21-28. doi: 10.1016/j. amjcard.2011.02.341

Torrents, C., \& Balague, N. (2006). Dynamic system theory and sports training. Education. Physical Training. Sport, 1(60), 72-82.

Tulppo, M. P., Hughson, R. L., Makikallio, T. H., Airaksinen, K. E., Seppanen, T., \& Huikuri, H. V. (2001). Effects of exercise and passive head-up tilt on fractal and complexity properties of heart rate dynamics. American Journal of Physiology. Heart and Circulatory Physiology, 280(3), H1081-1087.

Vainoras, A. (2002). Functional model of human organism reaction to load evaluation of sportsmen training effect. Education. Physical training. Sport, 44, 88-93. 